\title{
Tracheal motile cilia in mice require CAMSAP3 for formation of central microtubule pair and coordinated beating
}

\author{
Hiroko Saito ${ }^{1}$, Fumiko Matsukawa-Usami ${ }^{2}$, Toshihiko Fujimori², Toshiya \\ Kimura $^{1}$, Takahiro Ide ${ }^{3}$, Takaki Yamamoto ${ }^{4}$, Tatsuo Shibata ${ }^{5}$, Kenta Onoue ${ }^{6}$, \\ Satoko Okayama $^{6}$, Shigenobu Yonemura ${ }^{6}$, Kazuyo Misaki ${ }^{7}$, Yurina Soba ${ }^{8}$, Yasutaka \\ Kakui $^{8,9}$, Masamitsu Sato ${ }^{8}$, Mika Toya ${ }^{\mathbf{1 , 8 , 1 0}}$, and Masatoshi Takeichi ${ }^{1}$
}

${ }^{1}$ Laboratory for Cell Adhesion and Tissue Patterning, RIKEN Center for Biosystems Dynamics Research, 2-2-3 Minatojima-Minamimachi, Chuo-ku, Kobe 650-0047, Japan ${ }^{2}$ Division of Embryology, National Institute for Basic Biology, and Department of Basic Biology, School of Life Science, SOKENDAI, the Graduate University for Advanced Studies, 5-1 Higashiyama, Myodaiji-cho, Okazaki, 444-8787 Japan

${ }^{3}$ Laboratory for Organismal Patterning, RIKEN Center for Biosystems Dynamics Research, 2-2-3 Minatojima-Minamimachi, Chuo-ku, Kobe 650-0047, Japan ${ }^{4}$ Nonequilibrium Physics of Living Matter RIKEN Hakubi Research Team, RIKEN Center for Biosystems Dynamics Research, Kobe 650-0047, Japan ${ }^{5}$ Laboratory for Physical Biology, RIKEN Center for Biosystems Dynamics Research, 2-2-3 Minatojima-Minamimachi, Chuo-ku, Kobe 650-0047, Japan ${ }^{6}$ Laboratory for Ultrastructural Research, RIKEN Center for Biosystems Dynamics Research, 2-2-3 Minatojima-Minamimachi, Chuo-ku, Kobe 650-0047, Japan ${ }^{7}$ Ultrastructural Research Team, RIKEN Center for Life Science Technologies, 2-2-3 Minatojima-Minamimachi, Chuo-ku, Kobe 650-0047, Japan ${ }^{8}$ Laboratory of Cytoskeletal Logistics, Center for Advanced Biomedical Sciences (TWIns), Waseda University, 2-2 Wakamatsucho, Shinjuku-ku, Tokyo 1628480, Japan

${ }^{9}$ Waseda Institute for Advanced Study, Waseda University, 1-21-1

Nishiwaseda, Shinjuku-ku, Tokyo 169-0051, Japan

${ }^{10}$ Major in Bioscience, Global Center for Science and Engineering, Faculty of Science and Engineering, Waseda University, 3-4-1 Okubo, Shinjuku-ku, Tokyo 169-8555, Japan

Correspondence: Masatoshi Takeichi, RIKEN Center for Biosystems Dynamics Research, 2-2-3 Minatojima-Minamimachi, Chuo-ku, Kobe 650-0047, Japan. E-mail: masatoshi.takeichi@riken.jp. Phone: 81(78) 306-3116. Fax: 81(78) 306-3118. 


\begin{abstract}
Motile cilia of multiciliated epithelial cells undergo synchronized beating to produce fluid flow along the luminal surface of various organs. Each motile cilium consists of an axoneme and a basal body, which are linked by a 'transition zone'. The axoneme exhibits a characteristic 9+2 microtubule arrangement important for ciliary motion, but how this microtubule system is generated is not yet fully understood. Here we show that CAMSAP3, a protein that can stabilize the minus end of a microtubule, concentrates at multiple sites of the cilium-basal body complex, including the upper region of the transition zone or the axonemal basal plate where the central pair of microtubules (CP) initiates. CAMSAP3 dysfunction resulted in loss of the CP and partial distortion of the basal plate, as well as the failure of multicilia to undergo synchronized beating. These findings suggest that CAMSAP3 plays pivotal roles in the formation or stabilization of the $\mathrm{CP}$ by localizing at the basal region of the axoneme, and thereby supports the coordinated motion of multicilia in airway epithelial cells.
\end{abstract}

\title{
Introduction
}

Multiciliated cells play a central role in generating directional flow of fluids or particles over the surface of various epithelial tissues, such as oviduct epithelium, ependymal cells lining the brain ventricles, and the airway epithelium of the respiratory tract (Brooks and Wallingford, 2014). Motile cilia on these epithelial cells beat synchronously to produce hydrodynamic forces. Failure of cilia to undergo their 
coordinated movement causes various diseases, including a subgroup of primary ciliary dyskinesia (PCD) (Lee, 2011; Tilley et al., 2015; Damseh et al., 2017).

The individual motile cilium of multiciliated cells consists of a 9+2 microtubule arrangement, that is, nine microtubule doublets and a central pair of single microtubules (CP), which position at the outer and central zones, respectively. This core structure of a cilium, called an axoneme, is linked to the basal body (BB), a form of centriole, from which ciliogenesis begins during development (Dawe et al., 2007a). The connection between the axoneme and the BB is mediated by a zone called the 'transition zone (TZ)' (Czarnecki and Shah, 2012; Goncalves and Pelletier, 2017), and the CP sits just above the TZ, although the outer microtubules extend farther downward to join the triplet microtubules of the BB (Greenan et al., 2020). Although extensive studies have been conducted to understand how these ciliary structures form and how motile cilia move in a coordinated fashion (Satir and Christensen, 2007), the molecular mechanisms underlying these questions are still largely unresolved.

CP deficiency brings about defective motion of cilia or flagella (Smith and Yang, 2004; Dawe et al., 2007b; Lechtreck et al., 2008; Nozawa et al., 2013; Loreng and Smith, 2017), and is also associated with PCD (Bautista-Harris et al., 2000; Stannard et al., 2004; Burgoyne et al., 2014). For example, removal of the putative serine-threonine kinase Fused (Wilson et al., 2009; Nozawa et al., 2013) or radial spoke head proteins such as Rsph1 or Rsph9 (Kott et al., 2013; Zhu et al., 2019), results in partial or complete loss of $\mathrm{CP}$, simultaneously altering the ciliary beat pattern from planar to rotational in motile cilia. CP formation is thought to occur from TZ. Although 
molecular constituents of the TZ have been analyzed (Czarnecki and Shah, 2012; Dean et al., 2016), how the CP grows out from the $\mathrm{TZ}$ in motile cilia, however, remains unknown, particularly because no template for microtubule polymerization is detected at the TZ. In flagella, the CP is anchored to the "basal plate" at the distal end of the TZ. Recent studies identified "basalin' localized at the basal plate, and its depletion resulted in disruption of the basal plate as well as collapse of CP (Dean et al., 2019), suggesting that this structure is important for CP nucleation.

CAMSAP (calmodulin-regulated spectrin-associated protein) and its invertebrate homologs form a protein family, each member of which binds the minus end of noncentrosomal microtubules (Meng et al., 2008; Baines et al., 2009; Goodwin and Vale, 2010; Tanaka et al., 2012; Hendershott and Vale, 2014; Jiang et al., 2014; King et al., 2014; Richardson et al., 2014). Through this property of CAMSAP, it regulates the dynamics of the minus ends of microtubules, simultaneously anchoring them to particular subcellular sites (Nashchekin et al., 2016; Toya et al., 2016), and contributes to a variety of cell and tissue morphogenesis (Noordstra et al., 2016; Martin et al., 2018; Takeda et al., 2018; Ko et al., 2019; Kimura et al., 2021; Mitsuhata et al., 2021), including neuritegenesis (Chuang et al., 2014; Yau et al., 2014; Pongrakhananon et al., 2018; Feng et al., 2019; Wang et al., 2019; Chen et al., 2020).

The binding of CAMSAP to microtubules is mediated by its C-terminal CKK domain (Meng et al., 2008; Baines et al., 2009; Hendershott and Vale, 2014; Jiang et al., 2014), and its deletion causes the dysfunction of CAMSAP. For example, CAMSAP3, a member of this molecular family, is concentrated at the apical cortex in intestinal 
epithelial cells of mice through the action of the $\mathrm{CC} 1$ domain of the protein, tethering non-centrosomal microtubules to these sites, and this results in the assembly of microtubules whose plus ends point basally (Toya et al., 2016). This characteristic microtubule assembly is disrupted in mice bearing a mutant gene encoding a truncated Camsap3 (Camsap3 $3^{d c / d c}$ mice), in which the CKK domain is deleted, because mRNA transcribed from the mutated gene does not cover the exons that encode the CKK domain (Toya et al., 2016). Camsap $3^{d c / d c}$ mice show other defects, such as cyst formation in proximal renal tubules (Mitsuhata et al., 2021), and narrowing of the lateral ventricles in the brain (Kimura et al., 2021), although the overall brain architecture looks normal. Camsap $3^{d c / d c}$ mice and Camsap3 null mutant mice show similar abnormalities (Pongrakhananon et al., 2018; Mitsuhata et al., 2021), suggesting that CAMSAP3 lacking a CKK domain is fully non-functional.

A recent study showed that CAMSAP3 is expressed in nasal multiciliated cells, and its absence causes defects in ciliary motion and polarity (Robinson et al., 2020). Similar observations were also reported using oviduct cells (Usami et al., 2021). However, how CAMSAP3 sustains the structure and function of multicilia remains to be investigated. In the present study, we used airway epithelial cells of adult mice to elucidate the role of CAMSAP3 in coordinated movement of multicilia, finding that CAMSAP3 dysfunction due to CKK domain loss results in a partial disruption of axonemal structures at its proximal end, as well as loss of the CP. Superresolution microscopy revealed that a fraction of CAMSAP3 accumulates around the sites where the CP is supposed to be initiated. Based on these observations, we discuss potential mechanisms by which CAMSAP3 supports CP formation and coordinated motion of multicilia. 


\section{Results}

\section{Defective ciliary motion in Camsap3-mutated airway cells}

Camsap $3^{d c / d c}$ (Camsap3 mutant) mice frequently produced clicking or chattering sounds (Audio 1), suggesting that they might have respiratory problems. We examined the luminal surface of trachea collected from postnatal mice using scanning electronmicroscopy, finding that multiciliated epithelial cells are normally present in the trachea of the mutant mice, the distribution pattern of which was similar to that observed in wild-type trachea (Figure 1A). In higher magnification views, however, we noted that the alignment of multicilia along the longitudinal axis of trachea, which is generally well ordered in wild-type airway cells, was disorganized to some extent in Camsap3mutated cells (Figure 1B).

To examine whether the motile cilia of the mutant cells have any defects in motility, we isolated trachea and acquired time-lapse images of the cilia on airway epithelial cells. Wild-type cells exhibited a characteristic synchronized beating of multicilia (Video 1), whereas those of mutant cells tended to lose such orderly motion, instead displaying aberrant movements individually (Video 2). We then analyzed the videos to quantify the pattern of ciliary motion by calculating the flow field of a ciliary carpet using particle image velocimetry (PIV). In PIV analysis, we subdivide each image into smaller areas, and then quantify how fast and in which direction each area flows by calculating the cross-correlation between the subdivided areas in subsequent time frames. As a result, velocity is assigned to individual areas, and a flow field is obtained. The flow field for a wild-type cell showed that multiple cilia on the cell oscillated together collectively 
(Figure 1C and Video 3). In contrast, those in a mutant cell were subdivided into several smaller clusters, each of which showed a collective flow in a unique direction (Figure 1C and Video 4). Using these data, we quantified the degree of synchrony of ciliary motion by calculating the time-evolution of the average speed of the motion, which is the magnitude of the average velocity over the field of view. Since the individual cilia drive oscillatory motion in the flow field of PIV, if all ciliary motions are synchronized, the magnitude of the average velocity vector is expected to show oscillation. In contrast, if the ciliary motion is all spatially random, the magnitude of the average velocity vector would not oscillate in time. Our analysis showed that the magnitude of the average velocity vector oscillated less prominently in the mutant than in the wild-type cells, indicating that the degree of synchrony of ciliary motion was lower in the mutant cells (Figure 1D).

\section{Loss of the central microtubule pair in the axoneme of Camsap3-mutated motile} cilia

To investigate the structural basis underlying the uncoordinated beating of cilia in Camsap3-mutated cells, we used transmission electron microscopy. Longitudinal sections along the cilia revealed that the overall morphology of cilia and basal bodies looked normal in the mutants. However, the centrally located electron-dense signals that represent the $\mathrm{CP}$ were not clearly detectable in the mutant cells, as they were often discontinuous (Figure 2A). Cross sections of cilia revealed further details of such abnormalities. While the cilia in wild-type cells exhibit a typical $9+2$ arrangement of microtubules, any cilium of mutant cells did not have the CP. In these cells, 9 outer doublet microtubules were still present in $70 \%$ of cilia at the normal positions, but in the 
rest of them, one of the doublet microtubules was displaced to an inner zone of the axoneme (Figure 2B).

To seek the origin of these inner doublet microtubules, we reexamined the longitudinal sections and found that, when centrally located microtubules were detectable, they were tilted along the longitudinal axis, one end of which gets closer to peripheral regions of the axoneme around its basal positions (Figure 2C). These observations suggest the possibility that an outer microtubule doublet was abnormally internalized at upper regions of the axoneme, as observed in PCD (Burgoyne et al., 2014).

We then focused on the proximal zones of axonemes, where the CP is thought to be nucleated. In longitudinal sections, the $\mathrm{CP}$ ended at a zone characterized by the accumulation of electron-dense materials at around the outer microtubule doublets (Figure 2D left, dark arrow). As this zone positionally corresponds to the 'basal plate' or 'axonemal plate' that is observed in cilia of unicellular organisms and thought to be the site for CP nucleation (Tucker, 1971; Gilula and Satir, 1972; Dute and Kung, 1978; Hoog et al., 2014; Dean et al., 2016), we also call it 'basal plate (BP)', although this structure appears to have a cylindrical shape in the case of airway cells. Closer observations showed that the $\mathrm{CP}$ structure began at around the basal end of the $\mathrm{BP}$, but was often sticking out of this BP structure by $20 \mathrm{~nm}$ or so (Figure 2D, white arrow). Axonemes in Camsap3-mutated cells appeared to have a similar zone, except that microtubules were not always detectable in the BP of mutant samples, as seen in upper axonemal zones (Figure 2D right, dark arrow). 
To confirm these observations, we used transverse sections of an airway cell which are slightly tilted, as these allowed us to observe sequential changes in the inner structures of cilium and $\mathrm{BB}$ along the longitudinal axis within a single focal plane. In wild-type samples, we detected a group of ciliary sections, each of which exhibits an electrondense outer ring with a centrally located CP (Figure 2E, left), just as seen in the BP defined above, leading us to judge that this is a transverse view of the BP. In the cilia of Camsap3-mutated cells, this structure was deformed showing various abnormalities, such as missing CP (Figure 2E, BP1), overly condensed central materials (Figure 2E, BP2), and partial distortion of the outer ring (Figure 2E, BP3). Some of these BPs retained microtubules in the center (Figure 2E, BP4). Thus, not only the $\mathrm{CP}$ but also the entire BP architecture appeared abnormal in the mutant.

These sections also allowed us to examine the TZ, which is characterized by the presence of Y-shaped linkers. We did not find any difference in TZ structures between wild-type and mutant cilia, except that a small number of wild-type TZ contained CP (Figure 2E, BP-TZ); this sample probably represents a section at the boundary region of BP and TZ. The structure of the BBs also looked normal in Camsap3-mutated cells, although they tended to lose their rotational polarity in mutant cells, as assessed by measuring the direction of the 'basal foot' which normally faces the oral side in wildtype cells (Figure 2F).

\section{Concentration of CAMSAP3 around the proximal ends of cilia}

To begin investigating how CAMSAP3 contributes to microtubule assembly in axonemes, we observed its distribution in airway epithelial cells of adult trachea by 
immunofluorescence (IF) microscopy, using samples fixed with paraformaldehyde (PFA). The results showed that CAMSAP3 was concentrated around the apical plasma membranes (Figure 3A), as assessed by co-immunostaining for EZRIN, which is known to associate with the apical membranes or microvilli (Berryman et al., 1993), as observed in other epithelial cells (Toya et al., 2016; Robinson et al., 2020; Kimura et al., 2021; Usami et al., 2021). This position of CAMSAP3 corresponded to the proximal edge of strong $\alpha$-tubulin IF signals that should represent ciliary microtubules (Figure 3B). Weak CAMSAP3 IF stains were also detectable along cilia as well as in the cytoplasm. We then examined airway epithelia of Camsap3 mutant mice and found that the mutated CAMSAP3 molecules (CKK domain truncated CAMSAP3) expressed in the mice also accumulated at the apical zones, overlapping with EZRIN (Figure 3A) or ciliary microtubules at their proximal ends (Figure 3B). Curiously, however, the mutated molecule tended to diffuse into upper portions of cilia, even though its expression level was lower than that of the wild-type one (Figure 3C), suggesting that the truncated CAMSAP3 penetrates into the ciliary body more readily than the wildtype CAMSAP3.

\section{Localization of CAMSAP3 at three distinct sites}

To gain more detailed information on CAMSAP3 localization in the cilia and BBs, we co-immunostained CAMSAP3 and components of the cilium-BB complex, analyzing their IF images by Airyscan confocal laser-scanning microscopy, which can yield a 140 $\mathrm{nm}$ lateral resolution. For this experiment, we used methanol for fixation of tissues, since some of the proteins to be examined did not give reproducible IF signals in PFAfixed specimens. The analysis first revealed that CAMSAP3 IF signals, which are 
condensed at the apical zone of a cell, were split into at least three rows of CAMSAP3 puncta designated \#1,\#2 and \#3 (Figure 4A). The relative IF intensity of the puncta in one row to another row varied from sample to sample; an example of alternative CAMSAP3 patterns is shown in Figure S1. The puncta at the \#1 row tended to be variable in morphology, contrasted with a rounded appearance in other rows. Additional puncta were also detectable outside the three rows, but their presence or location was not fixed. Individual CAMSAP3 puncta comprising the three rows were also aligned along the apicobasal axis of the cell, likely due to their association with a single ciliumBB complex at different levels of the apicobasal axis. The distances (mean $\pm \mathrm{SD}$ ) between \#1 and \#2 and between \#2 and \#3 along the longitudinal axis were $476 \pm 126$ $\mathrm{nm}(\mathrm{n}=30) \mathrm{nm}$ and $364 \pm 47 \mathrm{~nm}(\mathrm{n}=34)$, respectively.

To determine the structural relationship between these CAMSAP3 puncta and the BB, we co-immunostained for CAMSAP3 and Odf2 which is known to localize at the transition fibers and basal feet (Kunimoto et al., 2012). Odf2 overlapped with the \#3 CAMSAP3 puncta at the upper portion of the former, further extending down to CAMSAP3-negative zones (Figure 4A, leftmost). $\gamma$-Tubulin, which has been reported to accumulate at the basal foot as well as at additional lower regions of BB in motile cilia (Hagiwara et al., 2000; Clare et al., 2014), was detected below the \#3 puncta, occasionally overlapping with faint CAMSAP3 IF signals located underneath the \#3 row (Figure 4A, middle). The distances (mean $\pm \mathrm{SD}$ ) between $\gamma$-tubulin and the \#1, \#2 or \#3 CAMSAP3 were estimated at $962 \pm 130(\mathrm{n}=25), 592 \pm 89 \mathrm{~nm}(\mathrm{n}=18)$ and $240 \pm$ $37 \mathrm{~nm}(\mathrm{n}=19)$, respectively (Figure 4A graph). 
We then detected microtubules by $\alpha$-tubulin immunostaining (Figure 4A, rightmost). Cytoplasmic microtubules formed meshwork below the \#3 CAMSAP3 puncta, and some of them entered into the \#3 zone, yielding their overlapping view. On the other hand, ciliary microtubules, which should have a structural continuity to the basal body, were not clearly detectable at their proximal regions in our methanol-fixed samples for unknown reasons. Nevertheless, CAMSAP3 puncta at the \#1 site exhibited some overlapping with microtubule clusters.

We next analyzed the distribution of the CKK domain-truncated CAMSAP3 by Airyscan microscopy in Camsap3-mutated cells (Figure 4B). As seen for wild-type CAMSAP3, the mutant molecules formed multiple rows. Co-immunostaining for CAMSAP3 and Odf2, $\gamma$-tubulin or $\alpha$-tubulin showed that their spatial relationships were similar to those found in wild-type cells. However, we could not convincingly detect the \#1 row, although CAMSAP3 IF signals were randomly present at the ciliary zone (Figure 4B). We also noted that about half of CAMSAP3 puncta in the \#2 row were missing. These suggest that, although the mutated CAMSAP3 still associates with the cilium-BB system at the \#3 site, it does not do so at the \#1 site, and its binding to the \#2 site also became unstable. The distance (mean $\pm \mathrm{SD}$ ) between the $\# 2$ and \#3 puncta was $335 \pm 46 \mathrm{~nm}(\mathrm{n}=23)$, and those between $\gamma$-tubulin and the $\# 2$ or $\# 3$ puncta were $674 \pm$ $96(\mathrm{n}=17)$ and $250 \pm 49 \mathrm{~nm}(\mathrm{n}=17)$, respectively (Figure 4B graph).

\section{Localization of CAMSAP3 puncta at the transition zone}

For more precise localization of CAMSAP3 puncta, we co-immunostained wild-type ciliated cells for CAMSAP3 and Chibby, a protein that localizes at a proximal region of 
TZ, playing a role in ciliogenesis (Enjolras et al., 2012; Burke et al., 2014). Confocal microscopy of longitudinal sections showed that Chibby localizes above the \#3 CAMSAP3 puncta (Figure 5A). We then used Airyscan microscopy, confirming that major Chibby puncta are located above the \#3 puncta. To relate the distribution of the \#2 and \#3 CAMSAP3 puncta with the actual structure of the cilia-BB complex, we overlaid their positions on its ultrastructural image, referring to the published location of $\gamma$-tubulin (Clare et al., 2014), Odf2 (Kunimoto et al., 2012) and Chibby (Burke et al., 2014) in motile cilia, which were determined at the ultrastructural levels (Figure 5B). This comparison of IF images with the ultrastructure suggests that the \#2 CAMSAP3 puncta are localized at a distal portion of $\mathrm{TZ}$ or at BP, while the \#3 CAMSAP3 puncta, at an upper region of the $\mathrm{BB}$.

Finally, we viewed the overall distribution pattern of basal bodies and microtubules from the top of ciliated cells in which GFP-tagged Centrin 2, a basal body protein, is genetically expressed (Higginbotham et al., 2004). Basal bodies exhibited typical horizontal arrays in wild-type cells, and a similar pattern was maintained in Camsap3mutated cells (Figure S2). Microtubules form a horizontal network at the inter-BB spaces (Kunimoto et al., 2012; Tateishi et al., 2013; Tateishi et al., 2017), and we did not find any particular change of this network in Camsap3-mutated cells (Figure S2, and Videos 5 and 6). Microtubules located below the basal bodies also formed networks, and their pattern was indistinguishable between wild-type and Camsap3mutated cells. Thus, it seems that Camsap 3 mutation did not affect the overall BB assembly, nor the horizontal networks of microtubules associated with the BBs. 


\section{Discussion}

The present study demonstrated that CAMSAP3 concentrates at multiple sites of the cilium-BB complex in tracheal motile cilia in mice, and dysfunction of this molecule results in unsynchronized beating of multicilia, as well as the loss of CP and other structural defects in this complex. Comparison of CAMSAP3 distribution with the ultrastructure of a cilium suggested that the \#2 site corresponds to BP or an upper region of TZ, and the \#1 and 3 sites are located more distal and proximal than the BP, respectively. A preceding study using nasal epithelial cells also reported that a Camsap3 hypomorphic mutation caused similar functional and structural defects in the multicilia (Robinson et al., 2020). This study, however, detected CAMSAP3 as a single dot located between the axoneme and BB in P30 mice, contrasted with our observation that the CAMSAP3-bearing region is subdivided into multiple sites. Two possibilities can be considered to explain the difference between the two observations. First, CAMSAP3 may interact with ciliary components in different ways between the nasal and tracheal multiciliated cells, and secondly, different sample preparation or microscopic methods can lead to different results. Actually, we noted a variation in the CAMSAP3 distribution pattern. Whichever the case is, the CAMSAP3 dot in P30 nasal cilia may correspond to the $\# 2$ or $\# 3$ puncta in tracheal cilia, considering their distance from $\gamma$ tubulin of the BB. In nasal motile cilia, an additional dot overlapping with $\gamma$-tubulin was also observed in P3 mice (Robinson et al., 2020), but we did not examine tracheal motile cilia in such young mice. 
The information that CAMSAP3 localizes around at the BP enabled us to discuss its potential role in CP formation on the basis of our knowledge about how CAMSAP3 controls microtubules. That is, it stabilizes the minus-end of a microtubule and simultaneously serves to tether the microtubule filament to particular subcellular sites (Nashchekin et al., 2016; Toya et al., 2016). Here, we propose that CAMSAP3 may function to stabilize the $\mathrm{CP}$ by binding to the minus end of microtubules at around the $\mathrm{BP}$, as the minus ends of axonemal microtubules are oriented basally (Euteneuer and Mcintosh, 1981). Removal of this mechanism would have led to a collapse of the CP. Notably, the CKK-truncated CAMSAP3 expressed in Camsap $3^{d c / d c}$ mice, which is unable to bind the minus-ends of microtubules, were still detected at the \#2 site but not always. It is possible that CAMSAP3 can stay at this site without binding microtubules, but its association with a putative binding partner became unstable under this situation, as observed in previous studies (Toya et al., 2016), and consistent with the idea that CAMSAP3 does interact with microtubules at this site. To test these ideas, however, it will be prerequisite to determine whether CAMSAP3 is located inside or outside the axoneme for its interaction with microtubules.

Since BP architecture was also perturbed in Camsap3-mutated airway cells, the presence of CAMSAP3 or CP may also play a role in maintaining this structure. On the other hand, detection of CAMSAP3 puncta at regions more distal than BP (at the \#1 site) suggest that CAMSAP3 may interact with microtubules at the axoneme, because its puncta disappeared in Camsap3-mutated cilia. This could be another site for CAMSAP3 to support the $\mathrm{CP}$, but its potential function remains to be further 
investigated. Mutated CAMSAP3 tended to diffuse into the upper regions of a cilium. This might have occurred due to the release of CAMSAP3 from the \#1 or \#2 sites.

Loss of CP may explain why the Camsap3-mutated motile cilia showed unsynchronized beating, since $\mathrm{CP}$ deficiency due to various mechanisms is known to coincide with disorganized ciliary movement (Wilson et al., 2009; Kott et al., 2013; Nozawa et al., 2013; Zhu et al., 2019). Defective CP may also have been involved in the perturbation of BB polarity observed in the absence of functional CAMSAP3. For example, the aberrant beating of multicilia in Camsap3-mutated cells cannot produce sufficient hydrodynamic forces that are required for the polarized alignment of them (Guirao et al., 2010). On the other hand, we cannot exclude the possibility that CAMSAP3 associated with the cilia-BB complex could have some direct role in determining BB polarity. Defects in this putative mechanism might have perturbed BB polarity and in turn induced uncoordinated beating of cilia.

What is the function of CAMSAP3 at the \#3 site that corresponds to the distal part of the BB? It overlaps with the upper component of Odf2 that is known to associate with transition fibers as well as basal feet, removal of which results in loss of BB but without affecting CP (Kunimoto et al., 2012). Despite their overlapping, CAMSAP3 mutants did not mimic what is observed in Odf2 mutants, suggesting that they are not functionally linked to one another. The loss of CAMSAP3 function did not affect the horizontal network of microtubules interspaced between BBs, suggesting that their assembly of microtubes is regulated by factors other than CAMSAP3, such as $\gamma$-tubulin, an authentic microtubule nucleator. Thus, the role of CAMSAP3 at the BB remains 
mysterious. It is noted that the CKK-deficient CAMSAP3 puncta were normally detectable at the \#3 site, implying that CAMSAP3 proteins cluster here independently of microtubules.

Curiously, the role of CAMSAP3 in multiciliated cells appears not thoroughly conserved among different organs, contrasted with many other ciliary components whose loss induces PCD. In multiciliated ependymal cells of the brain, CAMSAP3 does not particularly associate with the cilium-BB complex, and their ciliary beating occurs normally in Camsap3-mutant mice (Kimura et al., 2021). In the ependymal cells, CAMSAP3 localizes under the apical plasma membranes and its major role is to regulate the organization of cortical microtubules that are required for establishing a microtubule-dependent signaling system. These observations suggest that CAMSAP3 contributes to multiciliary organization in a tissue-specific manner, although the possibility that other CAMSAP subtypes or unidentified similar proteins have taken over the role of CAMSAP3 in ependymal cells is not excluded. It should also be noted that the CAMSAP family or CKK domain-containing-proteins have been detected only in the metazoan (Baines et al., 2009), which implies that their role in the ciliary system is limited to particular animal species. Future studies will be necessary to reveal how CAMSAP3 becomes associated with the cilium-BB complex and supports its structure and function in particular tissues and species.

\section{Materials and Methods}




\section{Mice}

A Camsap3 mutant mouse line, Camsap $3^{d c / d c}$, was described previously (Toya et al., 2016). The transgenic mouse line expressing GFP-Centrin2 (EGFP-CETN2) was a gift from Holden Higginbotham, Brigham Young University (Higginbotham et al., 2004). Female mice of this transgenic line were crossed with Camsap $^{{ }^{d c /+}}$ males to generate those expressing GFP-Centrin2 under Camsap3 mutation. ICR mice (Slc; ICR, Japan SLC) were used to for immunostaining analysis of Chibby. The age of mice used for the study was recorded for each experiment and described in the legends of figures. The experiments using mice were performed in accordance with the protocol(s) approved by the Institutional Animal Care and Use Committee of the RIKEN Center for Biosystems Dynamics Research (RIKEN Kobe Branch), or the Guidelines of Animal Experimentation of National Institutes for Natural Sciences.

\section{Scanning electron microscopy}

Mice were anesthetized with isoflurane, and dissected to reveal trachea and cut diaphragm. The tracheas were prefixed with a fixative solution $(2 \%$ paraformaldehyde and $2.5 \%$ glutaraldehyde in $0.1 \mathrm{M}$ cacodylate buffer, $\mathrm{pH} 7.4$ ) for $5 \mathrm{~min}$, then removed from mice. The trachea was cut into small pieces, and the samples were immersed in the same fixative solution at room temperature for $2 \mathrm{hr}$. The samples were washed with $0.1 \mathrm{M}$ cacodylate buffer and postfixed in $1 \% \mathrm{OsO} 4$ in $0.1 \mathrm{M}$ cacodylate buffer on ice for $2 \mathrm{hr}$. Then the samples were washed with distilled water, and stained overnight with $0.5 \%$ aqueous uranyl acetate at room temperature. The stained samples were dehydrated with ethanol the samples were rinsed with distilled water, and further stained with $0.5 \%$ uranyl acetate solution overnight at room temperature. The samples were rinsed with 
distilled water and dehydrated through a graded series of ethanol, transferred to isopentyl acetate and critical point-dried using liquid $\mathrm{CO}_{2}$. The dried specimens were evaporation-coated with Osmium and examined in a JSM-5600LV scanning electron microscope at an accelerating voltage of $10 \mathrm{kV}$.

\section{Transmission electron microscopy}

Tracheas were fixed with 2\% paraformaldehyde and $2.5 \%$ glutaraldehyde in $0.1 \mathrm{M}$ cacodylate buffer ( $\mathrm{pH} 7.4)$ at $4^{\circ} \mathrm{C}$ overnight. After washing five times with $0.1 \mathrm{M}$ cacodylate buffer, the specimens were further fixed with $1 \%$ OsO4 in $0.1 \mathrm{M}$ cacodylate buffer at $4^{\circ} \mathrm{C}$ for $1 \mathrm{~h}$ and then washed five times with Milli-Q water. The specimens were incubated in 1\% uranyl acetate overnight and washed five times with Milli-Q water. Specimens were dehydrated with ethanol series $(20 \%, 50 \%, 70 \%, 90 \%$ and $99.5 \%$ for $5 \mathrm{~min}$ each, and twice in $100 \%$ for $10 \mathrm{~min}$ ) and acetone, then followed by infiltration with Epon 812 (TAAB). Polymerization was performed at $60^{\circ} \mathrm{C}$ for $72 \mathrm{~h}$. Samples were cut with an ultramicrotome (ULTRA CUT UCT, Leica) equipped with a diamond knife (Diatome), doubly stained with uranyl acetate and lead citrate, and examined in a transmission electron microscope (JEM-1400 Plus ; JEOL) operated at $100 \mathrm{kV}$.

\section{Immunofluorescence staining and microscopy}

Isolated trachea tissue was fixed in a PEM buffer (PEM: 100 mM PIPES, 5 mM EGTA, and $5 \mathrm{mM} \mathrm{MgCl} 2$, adjusted to $\mathrm{pH} 6.9$ with $2 \mathrm{M} \mathrm{KOH}$ ) containing $2 \%$ paraformaldehyde and $0.1 \mathrm{M}$ sorbitol for $1 \mathrm{~h}$ at room temperature, except that the tissue was treated with $4 \%$ paraformaldehyde at $37^{\circ} \mathrm{C}$ for $1 \mathrm{hr}$ and then at $4{ }^{\circ} \mathrm{C}$ for $24 \mathrm{hr}$ for microtubule 
fixation. Alternatively, trachea tissue was fixed with methanol for 20 to $45 \mathrm{~min}$ at $20^{\circ} \mathrm{C}$, followed by treatment with acetone for $1 \mathrm{~min}$ at $-20^{\circ} \mathrm{C}$. After fixation, the tissue was rinsed three times with PEM for 10 min. Then, cryoprotection was carried out by incubation with $15 \%$ sucrose in PEM overnight at $4{ }^{\circ} \mathrm{C}$, followed by incubation with $20 \%$ sucrose in PEM for $6 \mathrm{~h}$, and $30 \%$ sucrose in PEM overnight at $4^{\circ} \mathrm{C}$. The tissue was frozen in OCT compound in an embedding mold (Polysciences, Inc.) in liquid nitrogen and stored at $-80^{\circ} \mathrm{C}$. Frozen samples were sliced at $7 \mathrm{~mm}$ thickness with a cryostat (HM500M, MICROM).

After washing twice with PBS, the sections were blocked with Blocking One (Nacalai Tesque) for $1 \mathrm{hr}$ at room temperature, followed by incubation with primary antibodies in Blocking One (Nacalai Tesque) overnight at $4^{\circ} \mathrm{C}$. Sections were washed two times with PBS and incubated for one hour at RT with corresponding secondary antibodies. Stained sections were washed two times with PBS and mounted with either Fluorsave (Chemicon) or 2,2'-thiodiethanol (Staudt et al., 2007). For samples used for Airyscan microscopy, antigen retrieval was performed by incubating sections in HistoVT One (Nacalai Tesque) for $3 \mathrm{~min}$ at $95^{\circ} \mathrm{C}$, prior to the incubation with primary antibodies.

For conventional immunofluorescence microscopy, samples were analyzed by Zeiss Axiopan 2 through Plan-APOCHROMAT 63x/1.4 Oil Dic or Plan-NEOFLUAR 40x/1.3 Oil Dic objectives. For confocal microscopy, a NIKON A1 microscope with Nikon Plan Apo Ve 100x/1.40 oil OFN25 DIC N2 objective lens was used. For superresolution microscopy, stacks of images were taken along the z-axis at optimal intervals using Airyscan (LSM880, Zeiss) with 63x/1.40 NA and a 100x/1.46 NA objective 
lenses. TCS SP8 STED with HCX PL APO CS 100x/1.40 oil immersion objective lens (Leica) was used to acquire the horizontal views of cells, and the optical conditions for this imaging are shown in Supplementary Table 1. Acquired images were processed using Zen (Zeiss), Adobe Photoshop (Adobe), or ImageJ (Wayne Rasband, NIH).

\section{Measurement of distances between CAMSAP3 puncta}

To determine the distance between two IF puncta, we measured from the center of one punctum to that of the other punctum, using ImageJ. Three to ten sections were used for each measurement.

\section{Quantification of ciliary orientation in multiciliated epithelial cells}

To quantify the alignment of multicilia along the longitudinal axis of trachea, the angles of cilia in SEM images were measured using ImageJ. Briefly, a line was drawn along individual cilia and then the angle of the line was measured. Measured angles of cilia were normalised by the average angle of all the cilia within the cell in order to eliminate a possible difference in the orientation of multiciliated epithelial cells. Polar plots for the distribution of ciliary angles were generated by ggplot2 in R. For the statistical test, we applied the F-test function (var.test) in R.

\section{Live imaging of ciliary motion and quantification of data}

Live images of cilia were acquired as described previously (Shinohara et al., 2015). Briefly, tracheas were collected into HEPES-buffered Dulbecco's modified Eagle's medium (pH7.2) supplemented with 10\% fetal bovine serum. Motility of cilia was examined at $25^{\circ} \mathrm{C}$ with a high-speed CMOS camera (HAS-U2, DITECT) that was 
connected to a microscope (BX53, Olympus) with a 40x objective lens (UPLFLN40X, Olympus) at a frame rate of 300 frames/s for tracheal cilia. To emphasize the movement of cilia, time-series images were adjusted to gain proper contrast and brightness after subtracting the minimum value through the images from each frame using ImageJ.

The synchrony of the motion of the cilia was quantified by calculating the flow field of the cilia carpet by PIV analysis. We denoised the time-lapse images (128 pix x 128 pix) by a Gaussian filter followed by the enhancement of the contrast using Fiji plugins. Then, the flow field of the time-lapse images was obtained by PIV analysis using a python library (OpenPIV). The interrogation window of PIV was 32 pix, and the overlap between adjacent interrogation areas was 24 pix.

\section{Antibodies}

The following primary antibodies were used: Rabbit anti-CAMSAP3 (Tanaka et al., 2012, 1: 100), mouse anti- $\alpha$-tubulin (clone DM1A, Sigma-Aldrich T9026, 1:1000), rabbit monoclonal anti- $\beta$-tubulin (clone 9F3, Cell Signaling Technology Cat\#2128, 1: 100), mouse anti- $\gamma$-tubulin (Sigma-Aldrich T6557, 1:1000), rabbit anti- $\gamma$-tubulin (Sigma-Aldrich T3559, 1:500), mouse anti-EZRIN (Abcam 4069, 1:100), rat anti-Odf2 (a gift from Sachiko Tsukita, Osaka University, 1: 2), mouse monoclonal anti-Chibby (Santa Cruz sc-101551, 1:100). The following secondary antibodies were used: Donkey CF-488A anti-rat IgG (Biotium 20027, 1:000) and CF-568 anti-rabbit IgG (Biotium 20698, 1:1000) antibodies; goat Alexa Fluor-488 anti-rat IgG (Invitrogen, A-11006, 1:500), Alexa Fluor-488 anti-rabbit IgG (Invitrogen, A-11034, 1:500), Alexa Fluor-568 anti-mouse IgG (Invitrogen, A-11031, 1:500), Alexa Fluor-594 anti-rabbit IgG 
(Invitrogen A-11037, 1:500), Alexa Fluor-647 anti-rabbit IgG (Invitrogen, A-21245, 1:500) and Alexa Fluor-647 anti-mouse IgG (Invitrogen, A-21236, 1:1000) antibodies; and goat STAR580 anti-mouse IgG (Abberior 2-0002-005-1, 1:500) and STAR635P anti-rat IgG (Abberior 2-0132-007-5, 1:500).

\section{Western blot analysis}

Trachea tissues were homogenized in TMEN buffer $(20 \mathrm{mM}$ Tris- $\mathrm{HCl} \mathrm{pH} 7.4,1 \mathrm{mM}$ $\mathrm{MgCl}_{2}, 2 \mathrm{mM}$ EDTA, and $150 \mathrm{mM} \mathrm{NaCl}$ ) containing the proteinase inhibitor cocktail cOmplete ultra (Roche, 5892970001) and the phosphatase inhibitor cocktail PhosSTOP (Roche, 4906845001). Then, 2\% (w/v) SDS solution was added to homogenized trachea tissues. Proteins were separated by 4-20\% gradient SDS-PAGE gel (Cosmobio, 414879) and then transferred to PVDF membrane (Millipore, IPVH00010). To detect CAMSAP3 and GAPDH for a loading control, anti-CAMSAP3 rabbit antibody $(1: 1,000)$ and anti-GAPDH mouse antibody (MBL, M171-3, 1:1,000) were used as primary antibodies. Quantification of band intensities was done using ImageJ.

\section{Audio recording}

Respiratory sound of mice was recorded using the Voice Memos application installed in iPhone XS (Apple).

\section{Acknowledgements}

We thank Hiroshi Hamada for his support of live imaging experiments, and Mitsuru Morimoto for his advice on the study of lung, Sachiko Tsukita for anti-Odf2 antibody, 
and Ritsu Kamiya for critical reading of the manuscript. This work was supported by the program Grant-in-Aid for Scientific Research (S) (grant number 25221104) from the Japan Society for Promotion of Science to M.Ta; the Japan Society for the Promotion of Science, KAKENHI (grant numbers 17H03689 and 16H06280) and the Japan Science and Technology Agency, Core Research for Evolutional Science and Technology (JPMJCR1654) to T.F.; the Japan Society for the Promotion of Science, KAKENHI (grant number JP20K06645) to M.To; and Daiichi Sankyo Foundation of Life Science to M.S.

\section{Author contributions}

Conceptualization: M.Ta., M.To.; Formal analysis: T.Y., T.S., Y.K.; Investigation: H.S., F.M-U., T.K., T.I., K.O., S.O., K.M., Y.S., Y.K., M.To.; Writing-original draft: M.Ta.; Writing-reviews \& editing: M.To., T.F., F.M-U.; Supervision: M.Ta., T.F., S.Y., M.S.; Project administration: M.Ta.; Funding acquisition: M.Ta., T.F. 


\section{References}

Baines, A.J., Bignone, P.A., King, M.D., Maggs, A.M., Bennett, P.M., Pinder, J.C., and Phillips, G.W. (2009). The CKK domain (DUF1781) binds microtubules and defines the CAMSAP/ssp4 family of animal proteins. Molecular biology and evolution 26, 20052014.

Bautista-Harris, G., Julia-Serda, G., Rodriguez de Castro, F., Santana-Benitez, I., and Cabrera-Navarro, P. (2000). Absence of central microtubules and transposition in the ciliary apparatus of three siblings. Respiration 67, 449-452.

Berryman, M., Franck, Z., and Bretscher, A. (1993). Ezrin is concentrated in the apical microvilli of a wide variety of epithelial cells whereas moesin is found primarily in endothelial cells. J Cell Sci 105 ( Pt 4), 1025-1043.

Brooks, E.R., and Wallingford, J.B. (2014). Multiciliated cells. Curr Biol 24, R973-982.

Burgoyne, T., Lewis, A., Dewar, A., Luther, P., Hogg, C., Shoemark, A., and Dixon, M. (2014). Characterizing the ultrastructure of primary ciliary dyskinesia transposition defect using electron tomography. Cytoskeleton (Hoboken) 71, 294-301.

Burke, M.C., Li, F.Q., Cyge, B., Arashiro, T., Brechbuhl, H.M., Chen, X., Siller, S.S., Weiss, M.A., O'Connell, C.B., Love, D., Westlake, C.J., Reynolds, S.D., Kuriyama, R., and Takemaru, K. (2014). Chibby promotes ciliary vesicle formation and basal body docking during airway cell differentiation. J Cell Biol 207, 123-137.

Chen, Y., Zheng, J., Li, X., Zhu, L., Shao, Z., Yan, X., and Zhu, X. (2020). Wdr47 Controls Neuronal Polarization through the Camsap Family Microtubule Minus-EndBinding Proteins. Cell Rep 31, 107526.

Chuang, M., Goncharov, A., Wang, S., Oegema, K., Jin, Y., and Chisholm, A.D. (2014). The microtubule minus-end-binding protein patronin/PTRN-1 is required for axon regeneration in C. elegans. Cell Rep 9, 874-883.

Clare, D.K., Magescas, J., Piolot, T., Dumoux, M., Vesque, C., Pichard, E., Dang, T., Duvauchelle, B., Poirier, F., and Delacour, D. (2014). Basal foot MTOC organizes pillar MTs required for coordination of beating cilia. Nat Commun 5, 4888. 
Czarnecki, P.G., and Shah, J.V. (2012). The ciliary transition zone: from morphology and molecules to medicine. Trends Cell Biol 22, 201-210.

Damseh, N., Quercia, N., Rumman, N., Dell, S.D., and Kim, R.H. (2017). Primary ciliary dyskinesia: mechanisms and management. Appl Clin Genet 10,67-74.

Dawe, H.R., Farr, H., and Gull, K. (2007a). Centriole/basal body morphogenesis and migration during ciliogenesis in animal cells. J Cell Sci 120, 7-15.

Dawe, H.R., Shaw, M.K., Farr, H., and Gull, K. (2007b). The hydrocephalus inducing gene product, Hydin, positions axonemal central pair microtubules. BMC Biol 5, 33 .

Dean, S., Moreira-Leite, F., and Gull, K. (2019). Basalin is an evolutionarily unconstrained protein revealed via a conserved role in flagellum basal plate function. Elife 8 .

Dean, S., Moreira-Leite, F., Varga, V., and Gull, K. (2016). Cilium transition zone proteome reveals compartmentalization and differential dynamics of ciliopathy complexes. Proc Natl Acad Sci U S A 113, E5135-5143.

Dute, R., and Kung, C. (1978). Ultrastructure of the proximal region of somatic cilia in Paramecium tetraurelia. J Cell Biol 78, 451-464.

Enjolras, C., Thomas, J., Chhin, B., Cortier, E., Duteyrat, J.L., Soulavie, F., Kernan, M.J., Laurencon, A., and Durand, B. (2012). Drosophila chibby is required for basal body formation and ciliogenesis but not for Wg signaling. J Cell Biol 197, 313-325.

Euteneuer, U., and Mcintosh, J.R. (1981). Polarity of Some Motility-Related Microtubules. P Natl Acad Sci-Biol 78, 372-376.

Feng, C., Thyagarajan, P., Shorey, M., Seebold, D.Y., Weiner, A.T., Albertson, R.M., Rao, K.S., Sagasti, A., Goetschius, D.J., and Rolls, M.M. (2019). Patronin-mediated minus end growth is required for dendritic microtubule polarity. J Cell Biol 218, 23092328 .

Gilula, N.B., and Satir, P. (1972). The ciliary necklace. A ciliary membrane specialization. J Cell Biol 53, 494-509.

Goncalves, J., and Pelletier, L. (2017). The Ciliary Transition Zone: Finding the Pieces and Assembling the Gate. Mol Cells 40, 243-253. 
Goodwin, S.S., and Vale, R.D. (2010). Patronin Regulates the Microtubule Network by Protecting Microtubule Minus Ends. Cell 143, 263-274.

Greenan, G.A., Vale, R.D., and Agard, D.A. (2020). Electron cryotomography of intact motile cilia defines the basal body to axoneme transition. J Cell Biol 219.

Guirao, B., Meunier, A., Mortaud, S., Aguilar, A., Corsi, J.M., Strehl, L., Hirota, Y., Desoeuvre, A., Boutin, C., Han, Y.G., Mirzadeh, Z., Cremer, H., Montcouquiol, M., Sawamoto, K., and Spassky, N. (2010). Coupling between hydrodynamic forces and planar cell polarity orients mammalian motile cilia. Nat Cell Biol 12, 341-350.

Hagiwara, H., Kano, A., Aoki, T., Ohwada, N., and Takata, K. (2000). Localization of gamma-tubulin to the basal foot associated with the basal body extending a cilium. Histochem J 32, 669-671.

Hendershott, M.C., and Vale, R.D. (2014). Regulation of microtubule minus-end dynamics by CAMSAPs and Patronin. Proc Natl Acad Sci U S A 111, 5860-5865.

Higginbotham, H., Bielas, S., Tanaka, T., and Gleeson, J.G. (2004). Transgenic mouse line with green-fluorescent protein-labeled Centrin 2 allows visualization of the centrosome in living cells. Transgenic Res 13, 155-164.

Hoog, J.L., Lacomble, S., O'Toole, E.T., Hoenger, A., McIntosh, J.R., and Gull, K. (2014). Modes of flagellar assembly in Chlamydomonas reinhardtii and Trypanosoma brucei. Elife 3, e01479.

Jiang, K., Hua, S., Mohan, R., Grigoriev, I., Yau, K.W., Liu, Q., Katrukha, E.A., Altelaar, A.F., Heck, A.J., Hoogenraad, C.C., and Akhmanova, A. (2014). Microtubule minus-end stabilization by polymerization-driven CAMSAP deposition. Dev Cell 28, 295-309.

Kimura, T., Saito, H., Kawasaki, M., and Takeichi, M. (2021). CAMSAP3 is required for mTORC1-dependent ependymal cell growth and lateral ventricle shaping in mouse brains. Development 148 .

King, M.D., Phillips, G.W., Bignone, P.A., Hayes, N.V., Pinder, J.C., and Baines, A.J. (2014). A conserved sequence in calmodulin regulated spectrin-associated protein 1 links its interaction with spectrin and calmodulin to neurite outgrowth. J Neurochem 128, 391-402. 
Ko, C.S., Tserunyan, V., and Martin, A.C. (2019). Microtubules promote intercellular contractile force transmission during tissue folding. J Cell Biol 218, 2726-2742.

Kott, E., Legendre, M., Copin, B., Papon, J.F., Dastot-Le Moal, F., Montantin, G., Duquesnoy, P., Piterboth, W., Amram, D., Bassinet, L., Beucher, J., Beydon, N., Deneuville, E., Houdouin, V., Journel, H., Just, J., Nathan, N., Tamalet, A., Collot, N., Jeanson, L., Le Gouez, M., Vallette, B., Vojtek, A.M., Epaud, R., Coste, A., Clement, A., Housset, B., Louis, B., Escudier, E., and Amselem, S. (2013). Loss-of-function mutations in RSPH1 cause primary ciliary dyskinesia with central-complex and radialspoke defects. Am J Hum Genet 93, 561-570.

Kunimoto, K., Yamazaki, Y., Nishida, T., Shinohara, K., Ishikawa, H., Hasegawa, T., Okanoue, T., Hamada, H., Noda, T., Tamura, A., Tsukita, S., and Tsukita, S. (2012). Coordinated ciliary beating requires Odf2-mediated polarization of basal bodies via basal feet. Cell 148, 189-200.

Lechtreck, K.F., Delmotte, P., Robinson, M.L., Sanderson, M.J., and Witman, G.B. (2008). Mutations in Hydin impair ciliary motility in mice. J Cell Biol 180, 633-643.

Lee, L. (2011). Mechanisms of mammalian ciliary motility: Insights from primary ciliary dyskinesia genetics. Gene 473, 57-66.

Loreng, T.D., and Smith, E.F. (2017). The Central Apparatus of Cilia and Eukaryotic Flagella. Cold Spring Harb Perspect Biol 9.

Martin, M., Veloso, A., Wu, J., Katrukha, E.A., and Akhmanova, A. (2018). Control of endothelial cell polarity and sprouting angiogenesis by non-centrosomal microtubules. Elife 7.

Meng, W., Mushika, Y., Ichii, T., and Takeichi, M. (2008). Anchorage of microtubule minus ends to adherens junctions regulates epithelial cell-cell contacts. Cell 135, 948959.

Mitsuhata, Y., Abe, T., Misaki, K., Nakajima, Y., Kiriya, K., Kawasaki, M., Kiyonari, H., Takeichi, M., Toya, M., and Sato, M. (2021). Cyst formation in proximal renal tubules caused by dysfunction of the microtubule minus-end regulator CAMSAP3. Sci Rep 11, 5857. 
Nashchekin, D., Fernandes, A.R., and St Johnston, D. (2016). Patronin/Shot Cortical Foci Assemble the Noncentrosomal Microtubule Array that Specifies the Drosophila Anterior-Posterior Axis. Dev Cell 38, 61-72.

Noordstra, I., Liu, Q., Nijenhuis, W., Hua, S., Jiang, K., Baars, M., Remmelzwaal, S., Martin, M., Kapitein, L.C., and Akhmanova, A. (2016). Control of apico-basal epithelial polarity by the microtubule minus-end-binding protein CAMSAP3 and spectraplakin ACF7. J Cell Sci 129, 4278-4288.

Nozawa, Y.I., Yao, E., Lin, C., Yang, J.H., Wilson, C.W., Gacayan, R., and Chuang, P.T. (2013). Fused (Stk36) is a ciliary protein required for central pair assembly and motile cilia orientation in the mammalian oviduct. Dev Dyn 242, 1307-1319.

Pongrakhananon, V., Saito, H., Hiver, S., Abe, T., Shioi, G., Meng, W., and Takeichi, M. (2018). CAMSAP3 maintains neuronal polarity through regulation of microtubule stability. Proc Natl Acad Sci U S A 115, 9750-9755.

Richardson, C.E., Spilker, K.A., Cueva, J.G., Perrino, J., Goodman, M.B., and Shen, K. (2014). PTRN-1, a microtubule minus end-binding CAMSAP homolog, promotes microtubule function in Caenorhabditis elegans neurons. Elife 3, e01498.

Robinson, A.M., Takahashi, S., Brotslaw, E.J., Ahmad, A., Ferrer, E., Procissi, D., Richter, C.P., Cheatham, M.A., Mitchell, B.J., and Zheng, J. (2020). CAMSAP3 facilitates basal body polarity and the formation of the central pair of microtubules in motile cilia. Proc Natl Acad Sci U S A 117, 13571-13579.

Satir, P., and Christensen, S.T. (2007). Overview of structure and function of mammalian cilia. Annu Rev Physiol 69, 377-400.

Shi, D., Komatsu, K., Hirao, M., Toyooka, Y., Koyama, H., Tissir, F., Goffinet, A.M., Uemura, T., and Fujimori, T. (2014). Celsrl is required for the generation of polarity at multiple levels of the mouse oviduct. Development 141, 4558-4568.

Shinohara, K., Chen, D., Nishida, T., Misaki, K., Yonemura, S., and Hamada, H. (2015). Absence of Radial Spokes in Mouse Node Cilia Is Required for Rotational Movement but Confers Ultrastructural Instability as a Trade-Off. Dev Cell 35, 236-246.

Smith, E.F., and Yang, P. (2004). The radial spokes and central apparatus: mechanochemical transducers that regulate flagellar motility. Cell Motil Cytoskeleton 57, 8-17. 
Stannard, W., Rutman, A., Wallis, C., and O'Callaghan, C. (2004). Central microtubular agenesis causing primary ciliary dyskinesia. Am J Respir Crit Care Med 169, 634-637.

Staudt, T., Lang, M.C., Medda, R., Engelhardt, J., and Hell, S.W. (2007). 2,2'thiodiethanol: a new water soluble mounting medium for high resolution optical microscopy. Microsc Res Tech 70, 1-9.

Takeda, M., Sami, M.M., and Wang, Y.C. (2018). A homeostatic apical microtubule network shortens cells for epithelial folding via a basal polarity shift. Nat Cell Biol 20, $36-45$.

Tanaka, N., Meng, W., Nagae, S., and Takeichi, M. (2012). Nezha/CAMSAP3 and CAMSAP2 cooperate in epithelial-specific organization of noncentrosomal microtubules. Proc Natl Acad Sci U S A 109, 20029-20034.

Tateishi, K., Nishida, T., Inoue, K., and Tsukita, S. (2017). Three-dimensional Organization of Layered Apical Cytoskeletal Networks Associated with Mouse Airway Tissue Development. Sci Rep 7, 43783.

Tateishi, K., Yamazaki, Y., Nishida, T., Watanabe, S., Kunimoto, K., Ishikawa, H., and Tsukita, S. (2013). Two appendages homologous between basal bodies and centrioles are formed using distinct Odf2 domains. J Cell Biol 203, 417-425.

Tilley, A.E., Walters, M.S., Shaykhiev, R., and Crystal, R.G. (2015). Cilia dysfunction in lung disease. Annu Rev Physiol 77, 379-406.

Toya, M., Kobayashi, S., Kawasaki, M., Shioi, G., Kaneko, M., Ishiuchi, T., Misaki, K., Meng, W., and Takeichi, M. (2016). CAMSAP3 orients the apical-to-basal polarity of microtubule arrays in epithelial cells. Proc Natl Acad Sci U S A 113, 332-337.

Tucker, J.B. (1971). Development and deployment of cilia, basal bodies, and other microtubular organelles in the cortex of the ciliate Nassula. J Cell Sci 9, 539-567.

Usami, F.M., Arata, M., Shi, D., Oka, S., Higuchi, Y., Tissir, F., Takeichi, M., and Fujimori, T. (2021). Intercellular and intracellular cilia orientation is coordinated by CELSR1 and CAMSAP3 in oviduct multi-ciliated cells. J Cell Sci 134. 
Wang, Y., Rui, M., Tang, Q., Bu, S., and Yu, F. (2019). Patronin governs minus-endout orientation of dendritic microtubules to promote dendrite pruning in Drosophila. Elife 8 .

Wilson, C.W., Nguyen, C.T., Chen, M.H., Yang, J.H., Gacayan, R., Huang, J., Chen, J.N., and Chuang, P.T. (2009). Fused has evolved divergent roles in vertebrate Hedgehog signalling and motile ciliogenesis. Nature 459, 98-102.

Yau, K.W., van Beuningen, S.F., Cunha-Ferreira, I., Cloin, B.M., van Battum, E.Y., Will, L., Schatzle, P., Tas, R.P., van Krugten, J., Katrukha, E.A., Jiang, K., Wulf, P.S., Mikhaylova, M., Harterink, M., Pasterkamp, R.J., Akhmanova, A., Kapitein, L.C., and Hoogenraad, C.C. (2014). Microtubule minus-end binding protein CAMSAP2 controls axon specification and dendrite development. Neuron 82, 1058-1073.

Zhu, L., Liu, H., Chen, Y., Yan, X., and Zhu, X. (2019). Rsph9 is critical for ciliary radial spoke assembly and central pair microtubule stability. Biol Cell 111, 29-38. 
Figure 1

A
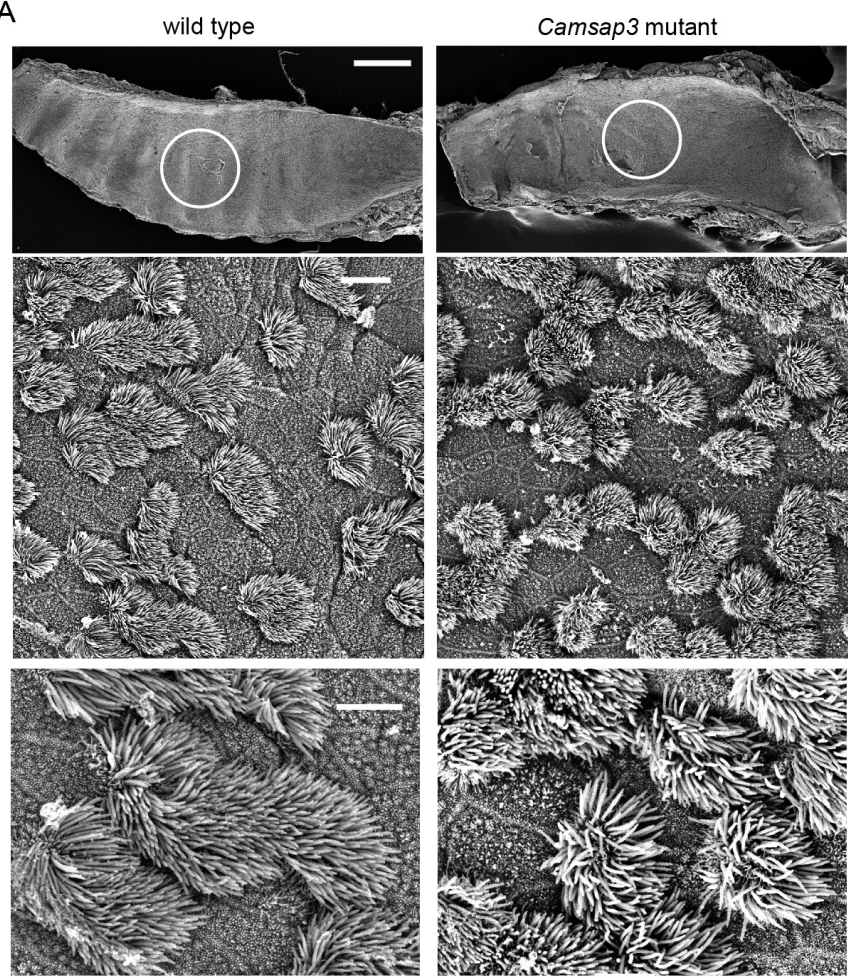

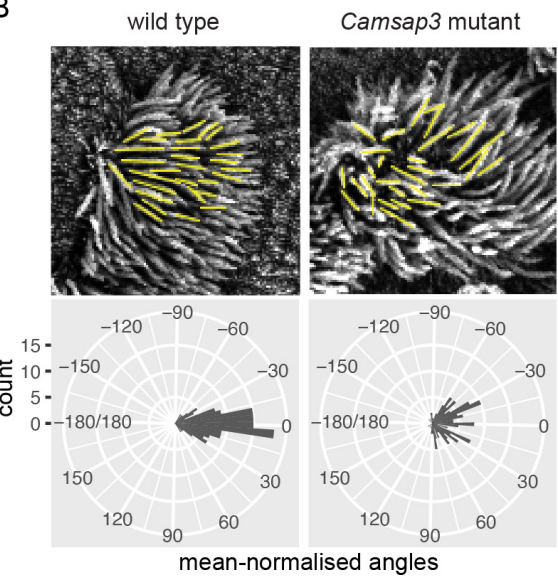

D

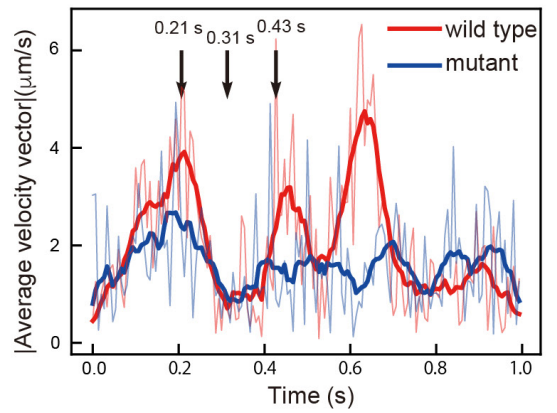

C

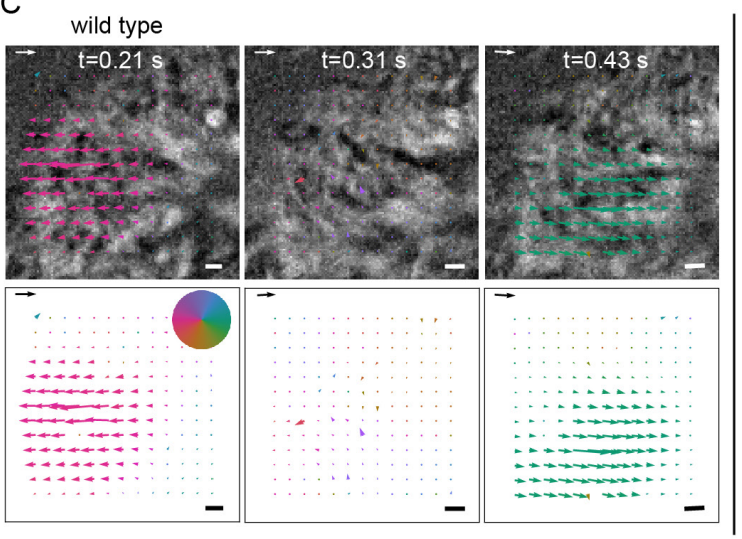

Camsap3 mutant

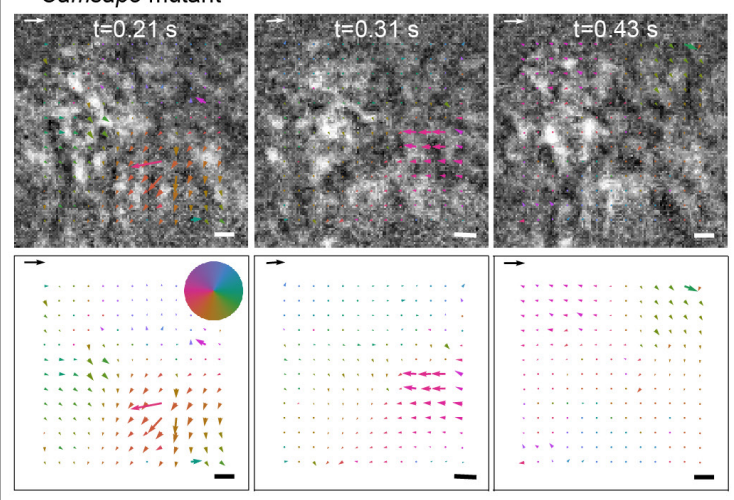

Figure 1. Morphology and movement of motile cilia in airway epithelial cells.

(A) Scanning electron microscopical observation of the luminal surface of trachea.

Trachea was isolated from P21 wild-type or Camsap3 mutant male mice. The top row shows a whole-mount trachea, in which the luminal surface is exposed. Part of the encircled region was enlarged in the middle and bottom rows. A representative image of 
three wild-type and five mutant samples is shown. Oral side is at the right. Scale bars: $500 \mu \mathrm{m}$, top; $10 \mu \mathrm{m}$, middle; and $5 \mu \mathrm{m}$, bottom.

(B) Quantitative analysis of ciliary orientation. Cilia were traced with yellow lines for angle measurement, and polar plots for the distribution of ciliary angles were obtained. The ciliary angles were normalized by the average angle in each cell. Angles of 30 cilia in five cells were measured and aggregated for the polar plots $\left(\mathrm{p}<2.2 \times 10^{-16}\right)$.

(C) Quantitative analysis of the collective motion of multicilia, which has been recorded in Videos 1 and 2, for a wild-type and Camsap3-mutated cell, respectively. These videos were used for PIV analysis (see Videos 3 and 4). Three snapshots, chosen from each video, are overlaid with the flow vector field calculated by PIV analysis, and only the vectors, which represent the velocity vectors, are shown at the bottom row. The color of the vectors indicates the angle of the velocity vector, and the colormap is shown as a pie chart. Scale arrow and scale bar on each image are $20 \mu \mathrm{m} / \mathrm{s}$ and $1 \mu \mathrm{m}$, respectively.

(D) Time-evolution of the magnitude of average velocity vector of ciliary motion plotted for wild-type (red curves) and mutant (blue curves) cells, respectively. The thin curves are the raw data, while the bold curves are the data smoothed by a moving average. The time points shown in B are indicated by black vertical arrows. 
A

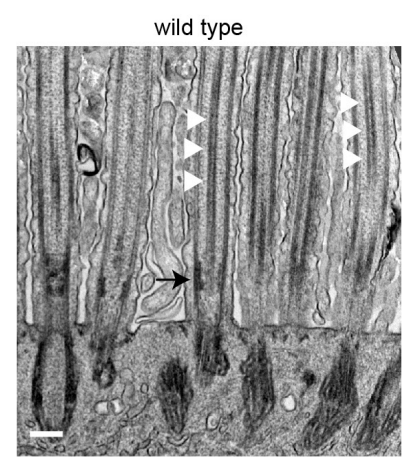

B

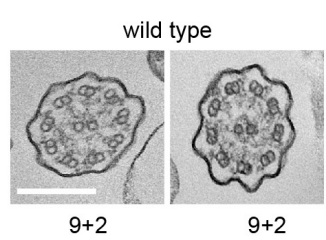

$\mathrm{E}$
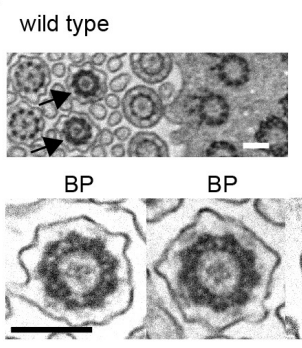

$\mathrm{F}$

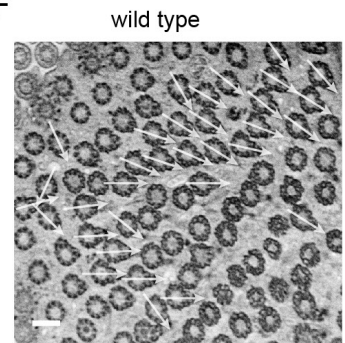

Camsap3 mutant
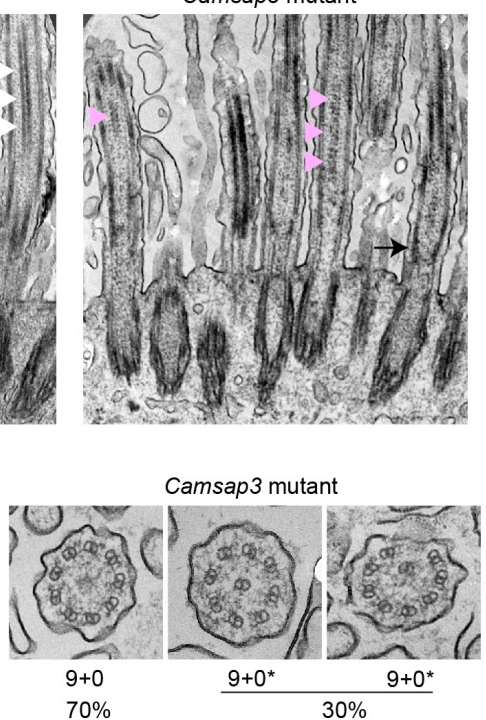

Camsap3 mutant
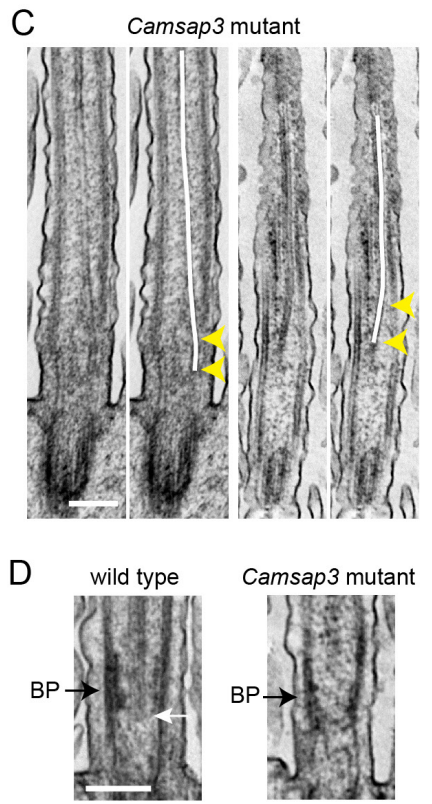
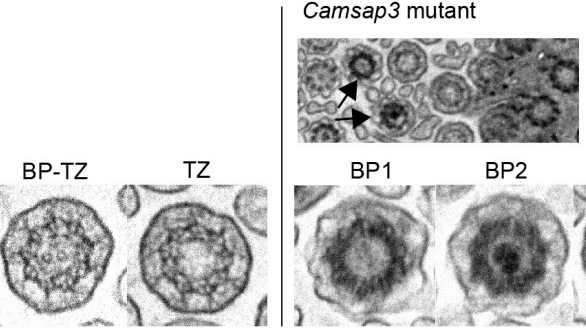

BP1

BP2

BP3

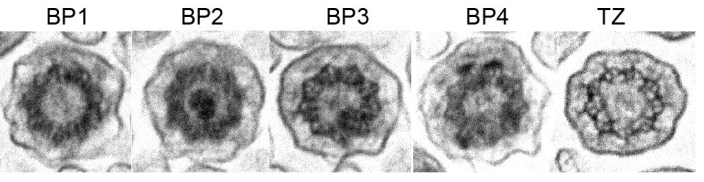

Camsap3 mutant
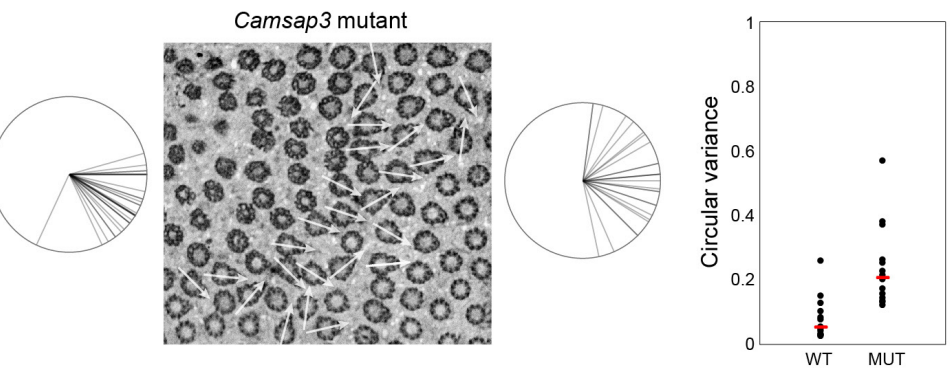

\section{Figure 2. Electron microscopic analysis of cilia and basal bodies.}

(A) Longitudinal section of a multiciliated cell, which yields a longitudinal view of axonemes and basal bodies. White triangles indicate examples of the CP in a wild-type cell; magenta triangles point to intermittent structures at the central position of axoneme in a Camsap3-mutated cell. Black arrows point to the 'basal plate', and a corresponding 
position in the Camsap 3 mutant sample. A representative image of more than 50 sections is shown for each genotype.

(B) Transverse section of cilia. In the mutant samples, 218 cilia from two independent sections were used for analysis. The mutant axonemes did not contain a CP, instead some of them (marked as $9+0^{*}$ ) had a doublet microtubule that was mislocalized to central or semi-central positions.

(C) Longitudinal section of cilia in Camsap3-mutated cells. A centrally located microtubule structure tilts toward the peripheral zone at basal portions of axoneme, as indicated by yellow arrowheads. Two examples are shown, each of which is duplicated to trace the central microtubule with a white line over the image.

(D) Part of the image in $\mathrm{A}$ is enlarged. White arrow points to the proximal end of the CP. BP, basal plate.

(E) Slightly oblique, transverse sections of a multi-ciliated cell, showing cross-sectional views of axoneme and $\mathrm{BB}$ at various longitudinal levels, in which arrows point to BPs that are characterized by a dense peripheral ring. Typical examples of ciliary sections at the level of BP and TZ are enlarged at the bottom. The image labeled BP-TZ likely represents a section at the boundary between $\mathrm{BP}$ and $\mathrm{TZ}$. In the mutant sections, all of the BPs show some abnormality, including loss or over-condensation of the central structure, and partial deformation of the peripheral ring. Typical examples are shown. More than 20 sections, each of which contains more than 50 cilia, were examined for each genotype.

(F) Slightly oblique, transverse section of a multi-ciliated cell, focusing on the basal foot of basal bodies. The direction of individual basal feet is shown with white arrows on the image, and these are aggregated at the right of each image. The range of orientation of basal feet on the cell varied from $26^{\circ}$ to $108^{\circ}$ in the wild type, with one exceptional case, and $13^{\circ}$ to $172^{\circ}$ in the mutant sample. We counted 33 and 26 basal bodies for the wild-type and mutant samples, respectively. Graph shows the variation in basal foot polarity in 13 wild-type or 14 mutant cells, which was quantified using circular variance (CV) that was defined previously (Shi et al., 2014), where cells with lower CV represent uniform BB orientation. Red bar, median. WT, wild type; MUT, Camsap3 mutant.

Age of mice used, P126. Thickness of sections, 50 to $70 \mathrm{~nm}$. Scale bars, $200 \mathrm{~nm}$ in A-E; $400 \mathrm{~nm}$ in $\mathrm{F}$. 
A CAMSAP3 EZRIN
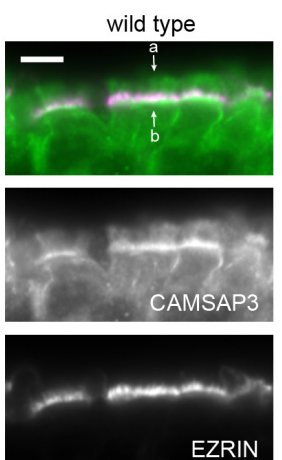

EZRIN

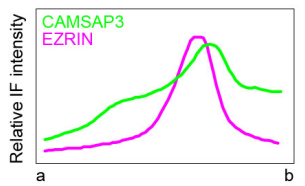

C
Camsap3 mutant
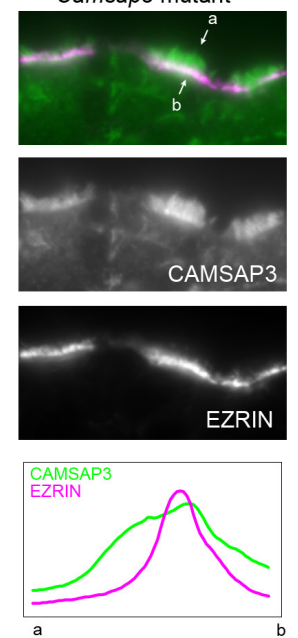

B CAMSAP3 2 -tubulin
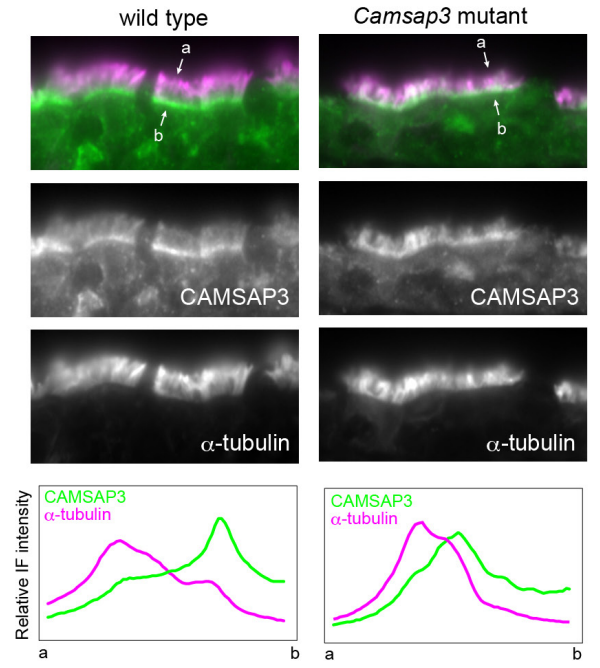

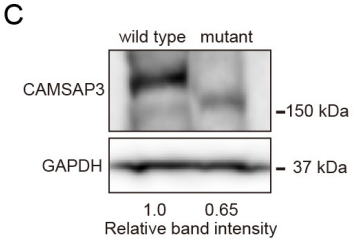

Figure 3. Immunostaining localization of CAMSAP3 in tracheal epithelium.

(A, B) Trachea derived from P176 mice were fixed with 2\% PFA, and processed for coimmunostaining for CAMSAP3 and EZRIN (A) or $\alpha$-tubulin (B). Images were recorded by conventional immunofluorescence microscopy. Immunofluorescence signals were scanned along the line (not shown) drawn between the two arrows, across the cilia-BB complex from its apical (a) to basal (b) side. A typical image of three to five sections is shown for each set of immunostaining. Scale bars, $10 \mu \mathrm{m}$.

(C) Western blots for CAMSAP3 in trachea isolated from P172 wild-type or Camsap $3^{d c / d c}$ mice. 
A wild type

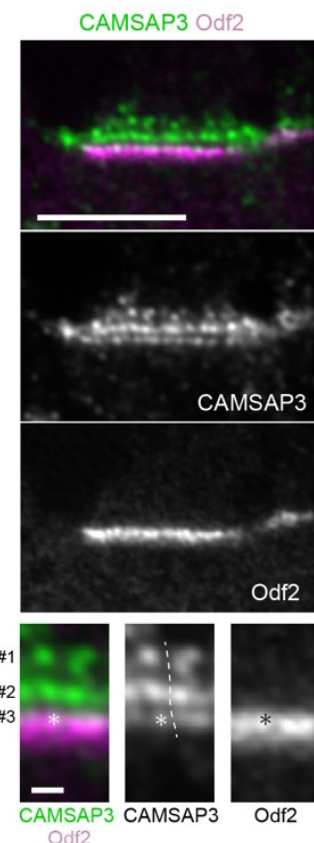

B Camsap3 mutant
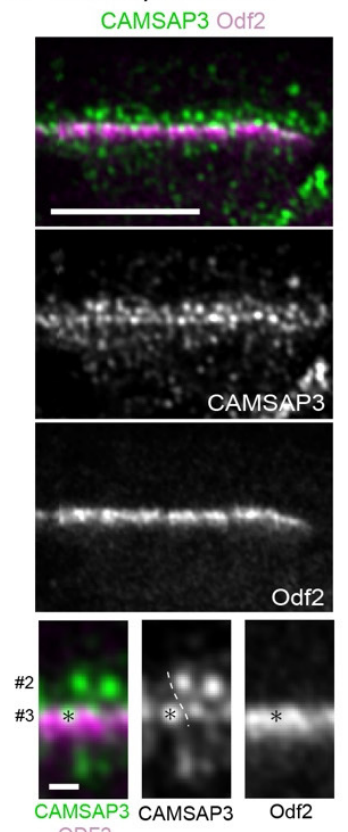

CAMSAP3 $\gamma-$-tubulin
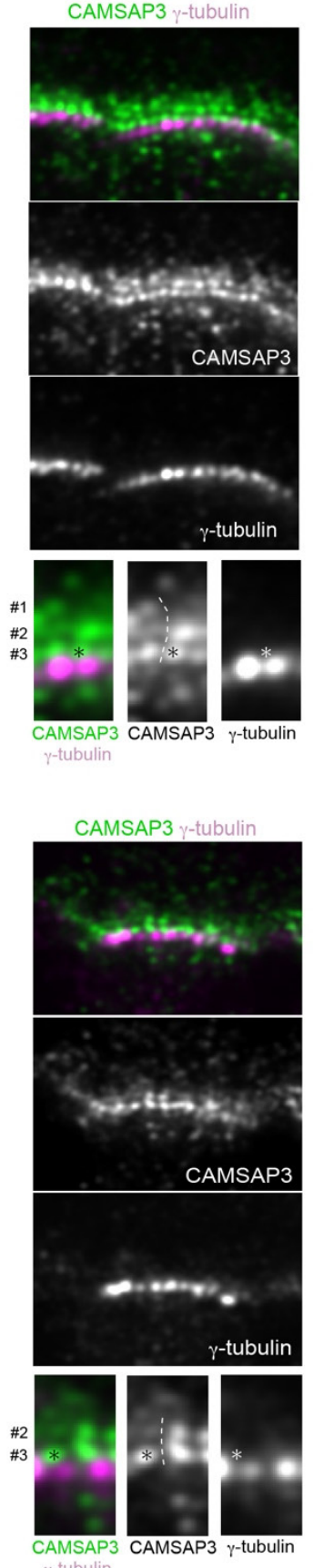

CAMSAP3 3 -tubulin

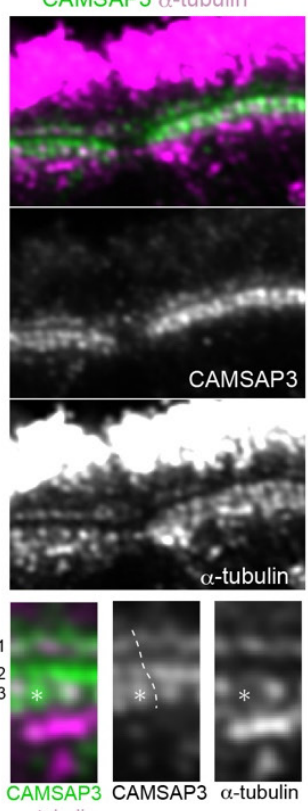

a-tubulin

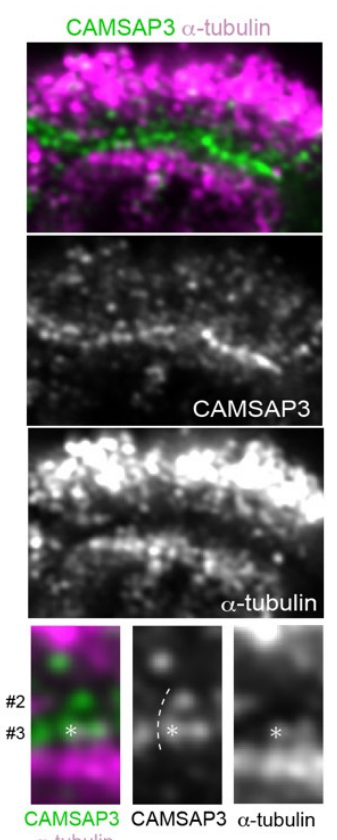

Figure 4

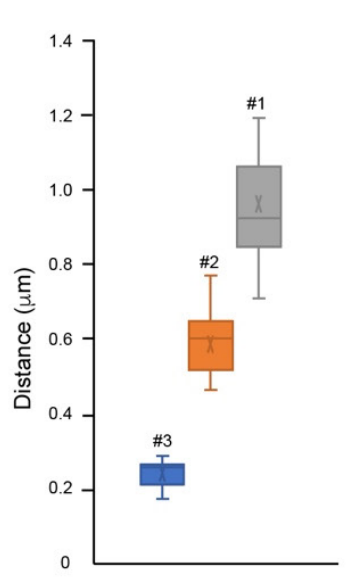

Figure 4. Airyscan microscopic analysis of CAMSAP3 distribution in wild-type and Camsap3-mutated multi-ciliated cells.

Longitudinal section of multi-ciliated cells in wild-type (A) or Camsap3-mutated (B) trachea, which were co-immunostained for the molecules indicated and recorded by Airyscan microscopy. Part of each image was enlarged at the bottom, in which the numbers indicate three different sites of CAMSAP3 puncta. Asterisks mark an example 
of CAMSAP3 puncta (or their positions) at the \#3 site in each image. Dotted lines indicate a putative boundary between the two sets of CAMSAP3 puncta aligned along the apicobasal axis. A typical image was selected from 8 to 10 sections of tissues, each of which contain several cells that can be analyzed, for each set of immunostaining. The samples were fixed with methanol. The graphs show the distance of CAMSAP3 puncta at different rows from $\gamma$-tubulin puncta. P91, P86 and P176 mice were used for immunostaining for Odf2, $\gamma$-tubulin, and $\alpha$-tubulin, respectively.

Scale bars, 5 and $0.5 \mu \mathrm{m}$ in the original and enlarged images, respectively. 
A

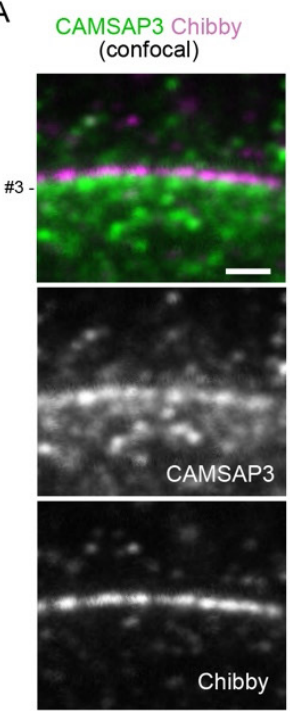

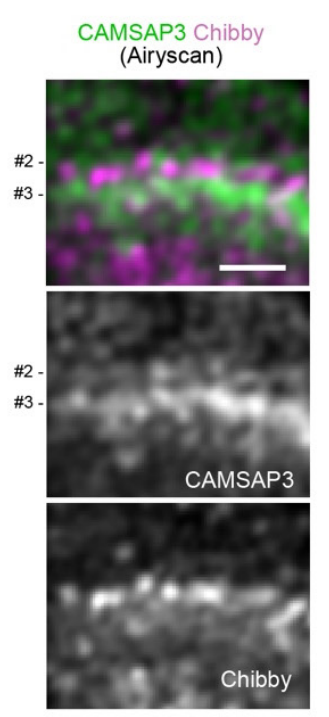

B

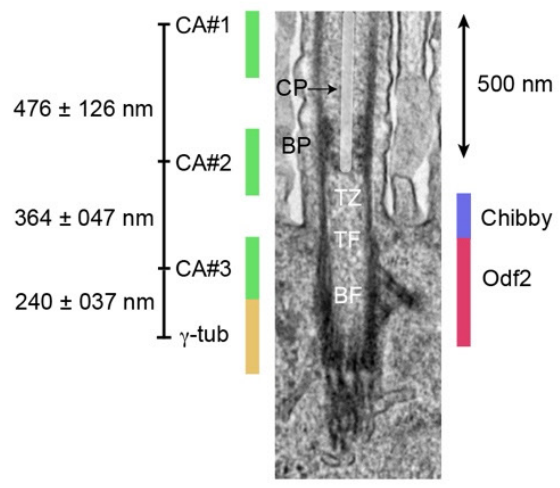

Figure 5. CAMASP3 localization in the transition zone.

(A) Longitudinal section of a wild-type multiciliated cell, co-immunostained for CAMSAP3 and Chibby, and recorded by confocal or Airyscan microscopy. In these samples, the relative IF intensity of \#3 CAMSAP3 puncta is high. Trachea were collected from P180 mice and fixed with methanol. Scale bar, $1 \mu \mathrm{m}$.

(B) A putative map of CAMSAP3 puncta in relation to the ultrastructure of the axoneme, transition zone and basal body. The relative position of each CAMSAP3 punctum was determined by measuring the distances between the puncta, and then the position of the entire CAMSAP3 puncta relative to the ultrastructure was adjusted by placing the \#3 punctum at the level of the upper half of Odf2 distribution, as observed in Figure 4. The positions of Chibby, Odf 2 and $\gamma$-tubulin were estimated referring to previous publications, although $\gamma$-tubulin localization needs further confirmation. The scale of the ultrastructure image is shown by a $500 \mathrm{~nm}$ bar. The CP is overlaid with a semitransparent white color. CP, the central pair of microtubules; BP, basal plate; TZ, transition zone; TF, transition fiber; BF, basal foot; $\gamma$-tub, $\gamma$-tubulin; CA, CAMSAP3. 
Figure S1

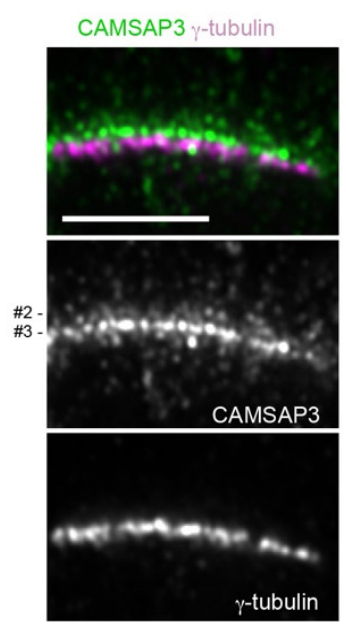

Supplementary Figure S1. An alternative pattern of CAMSAP3 distribution.

Longitudinal section of a multi-ciliated cell in wild-type trachea, which was coimmunostained for CAMSAP3 and $\gamma$-tubulin and recorded by Airyscan microscopy.

Note that the $\# 3$ row, identified by its position next to $\gamma$-tubulin, shows the most intense IF signals in this particular specimen. 

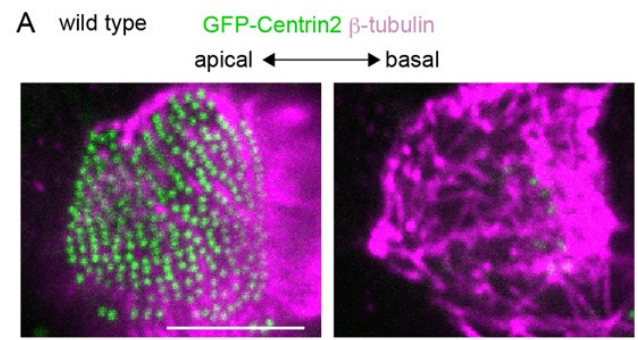

B Camsap3 mutant GFP-Centrin2 $\beta$-tubulin
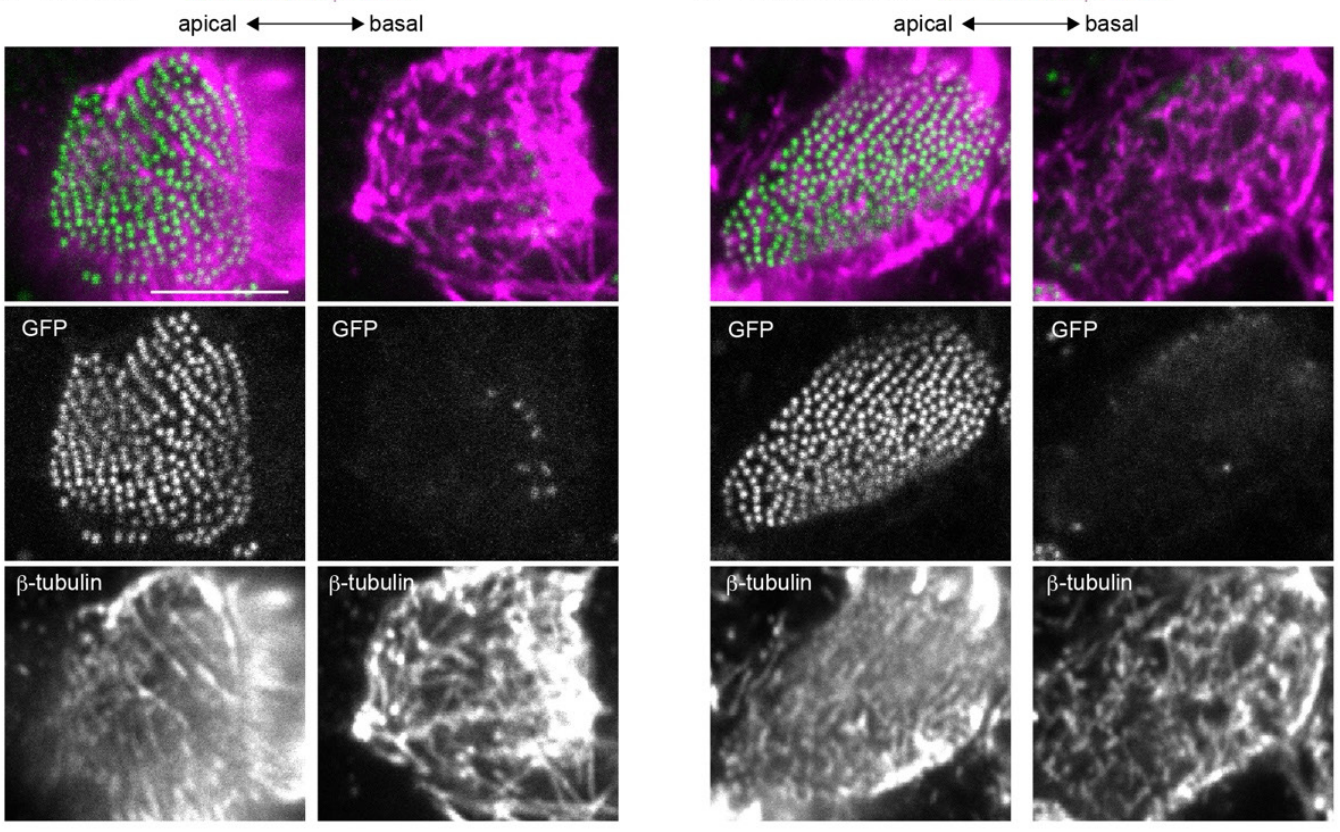

\section{Supplementary Figure S2. Microtubule distribution at the apical portions of a}

multiciliated cell. $\beta$-tubulin was immunostained using trachea isolated from a wild-type (A) or Camsap $3^{d c / d c}(\mathrm{~B})$ mouse expressing GFP-Centrin2. The images at two horizontal levels, which are 0.12 (in A) and $0.10 \mu \mathrm{m}$ (in B) apart, are shown. Oral side is at the right. P180 mice were used. The same samples were used in Videos 5 and 6.

Scale bars, $5 \mu \mathrm{m}$. 


\section{Video Legends}

Video 1. Beating multicilia in a wild-type airway epithelial cell. The time-lapse images were acquired at $300 \mathrm{fps}$ and displayed at $31 \mathrm{fps}$.

Video 2. Beating multicilia in a Camsap3-mutated airway epithelial cell. The time-lapse images were acquired at $300 \mathrm{fps}$ and displayed at $31 \mathrm{fps}$.

Video 3. Beating multicilia in a wild-type airway epithelial cell, which is overlaid with a flow vector field calculated by PIV analysis. The flow vector field was also shown separately at the right. The scale arrow and scale bar are $20 \mu \mathrm{m} / \mathrm{s}$ and $1 \mu \mathrm{m}$, respectively. Part of Video 1 was used for this analysis.

Video 4. Beating multicilia in a Camsap3-mutated airway epithelial cell, which is overlaid with a flow vector field calculated by PIV analysis. The flow vector field was also shown separately at the right. The scale arrow and scale bar are $20 \mu \mathrm{m} / \mathrm{s}$ and $1 \mu \mathrm{m}$, respectively. Part of Video 2 was used for this analysis.

Video 5. Animation of sequential optical sections, each of which is $0.2 \mu \mathrm{m}$ thick, of a wild-type multi-ciliated epithelial cell, in which GFP-Centrin (green) and $\beta$-tubulin (magenta) are visualized by fluorescence signals. The animation view begins around the level where the array of GFP-Centrin is detectable, then shifts toward a more basal view of the cell at a speed of $2 \mathrm{fps}$. See also Figure $6 \mathrm{~A}$.

Video 6. Animation of sequential optical sections, each of which is $0.2 \mu \mathrm{m}$ thick, of a Camsap3-mutated multi-ciliated epithelial cell, in which GFP-Centrin (green) and $\beta$ tubulin (magenta) are visualized by fluorescence signals. The animation view begins at the level where the array of GFP-Centrin is detectable, then shifts toward a more basal view of the cell at a speed of $2 \mathrm{fps}$. See also Figure 6B.

\section{Audio Legends}

Audio 1. Respiratory sound of a P50 Camsap3 mutant mouse. 